\title{
同顺序 $\boldsymbol{m} \times \boldsymbol{n}$ 排序问题的一个新方法
}

\author{
越民义韩继业 \\ (中国科学院数学研究所)
}

排序问题是一类很广泛的组合最优化问题. 它出现在工业、农业、运输和计划管理等方 面. 它主要研究一些服务者应按照怎样的次序去服务若干个被服务者, 以使预先给定的目标 函数达到最优. 文献中一般称服务者为 “机器”, 称被服务者为 “工件”。同顺序 $m \times n$ 排序 问题是指:

（1） $n$ 个工件皆依同一顺序 $\left(M_{1} 、 M_{2} \cdots M_{m}\right)$ 通过 $m$ 个机器；

（2）各工件在各机器上的加工时间为已知,且为常数;

(3) 一个机器只能同时加工一个工件;

(4) 在各机器上工件的加工次序都一样.

一般以总加工时间作为 $m \times n$ 排序问题的目标函数. 所谓总加工时间是指从机器 $M_{1}$ 开 始加工工件起, 到机器 $M_{m}$ 加工完全部工件为止这段时间. 显然它依赖于工件的加工次序.最 优加工次序(或工件的最优排列)对应的总加工时间最短.

文献[1]证明了当 $m \geqslant 3$ 时 $m \times n$ 排序问题属于“ $N P$ 完备”问题.目前解决 $m \times n$ 排序问题的 方法有分支定界法和消去法. 前者的主要成果被总结在文献[2]中; 后者的最新成果是 Szwarc 的消去准则 ${ }^{[3,4]}$, 他证明他的消去准则比文献中已有的结果都广泛. 在本文中我们给出几个消 去准则, 并证明我们的结果比 Szwarc 的结果要广泛; 其次我们给出计算最优加工次序的新方 法,通过实际计算,它比已有的方法要好.

\section{一、几个消去准则}

先定义几个符号:

1) $N=\{1,2,3, \cdots, n\}$ 是全部工件号码的集合.

2) $S=\left(s_{1} s_{2} \cdots s_{k}\right)$ 是任意 $k$ 个工件 $s_{1}, s_{2}, \cdots, s_{k}$ 排成的一个序列, 整数 $p$ 和 $q$ 满足 $1 \leqslant$ $p \leqslant q \leqslant m$, 我们令 $t_{p q}(S)$ 表示矩阵

$$
\left[\begin{array}{cccc}
a_{p s_{1}} & a_{p s_{2}} \cdots a_{p s_{k}} \\
a_{p+1 s_{1}} & a_{p+1 s_{1}} \cdots a_{p+1 s_{k}} \\
\cdots & \cdots \cdots \cdots \\
a_{q s_{1}} & a_{q s_{2}} \cdots a_{q c_{k}}
\end{array}\right]
$$

的最大可行和(最大可行和的定义见文献 [2]), 其中 $a_{p s}$ 是工件 $s$ 在机器 $M_{p}$ 上的加工时间. 当 $S$ 为空集时, 令 $t_{p q}(S)=0$. 
3) 设 $S$ 和 $S^{\prime}$ 是工件的序列, 且 $S \cap S^{\prime}=\phi$. 我们令 $B\left(S \cdots S^{\prime}\right)$ 表示全部 $\left(S \cdots S^{\prime}\right)$ 形的排 列(或加工次序)对应的总加工时间的下界. $S$ 或 $S^{\prime}$ 可为空集.

4) 设 $I=\left(i_{1} i_{2} \cdots i_{k}\right)$ 是工件的序列, $p$ 和 $q$ 为整数, $1 \leqslant p \leqslant q \leqslant m$, 我们令

$$
A_{p q}(I)=\min _{p \leqslant r_{1} \leqslant r_{2} \leqslant \cdots \leqslant r_{b} \leqslant q}\left(a_{r_{1} i_{1}}+a_{r_{2} z_{2}}+\cdots+a_{r_{k} i_{k}}\right) .
$$

5) 设 $S=\left(s_{1} s_{2} \cdots\right), I=\left(i_{1} i_{2} \cdots\right)$ 是工件的序列，我们令 $(S j)$ 表序列 $\left(s_{1} s_{2} \cdots j\right),(S I j)$ 表序列 $\left(s_{1} s_{2} \cdots i_{1} i_{i} \cdots \cdot j\right)$.

下面的定理说明: 当我们已排了序列 $s$ 后,工件 $j$ 可否排在下一个位置上.

定理 1 设 $I=\left(i_{1} i_{2} \cdots i_{u}\right), S=\left(s_{1} s_{2} \cdots s_{k}\right), I \cap S=\phi, j$ 不属于 $I$ 和 $S$, 序列集 $Q$ 是 $S$, $I, i$ 以外的所有工件所排的序列的全体。若条件

$$
\begin{array}{r}
t_{1 q}(S I) \leqslant \max \left\{t_{1 q}(S j)-a_{q j}+A_{q m}(I), B(S j \cdots)-\max _{R \in Q} t_{q m}(j R)\right\}, \\
1 \leqslant q \leqslant m
\end{array}
$$

成立，则在求最优排列时可消去所有 $\left(S j \cdots i_{1} \cdots i_{2} \cdots i_{u} \cdots\right)$ 形的排列，而不会把最优排列全 消光.

系 1 、设 $I=(i)$, 若条件

$t_{1 q}(S i) \leqslant \max \left\{t_{1 q}(S j)-a_{q j}+\min \left(a_{q i}, a_{q+1 i}, \cdots, a_{m i}\right), B(S j \cdots)-\max _{R \in Q} t_{q m}^{\tau_{m}}(j R)\right\}$,

$$
1 \leqslant q \leqslant m
$$

成立,则可消去所有 $(S j \cdots)$ 形的排列, 而不会把最优排列全消光.

Szwarc 在文献 [4] 中给出如下定理:

设 $S, R^{\prime}, R^{\prime \prime}$ 都是工件排成的序列, 它们满足

$$
R^{\prime} \cap R^{\prime \prime}=\phi, \quad\left\{R^{\prime} \cup R^{\prime \prime}\right\} \cap\left(S_{i j}\right)=\phi, \quad R^{\prime} \cup R^{\prime \prime} \cup(S i j)=N,
$$

工件 $i, j$ 不属于 $S$. 则条件

和条件

$$
t_{1 q-1}(S i j)-t_{1 q-1}(S j) \leqslant t_{1 q}(S i j)-t_{1 q}(S i) \leqslant a_{q i}, q=2, \cdots, m
$$

$$
\boldsymbol{t}_{1 m}\left(S_{i j} R^{\prime} R^{\prime \prime}\right) \leqslant t_{1 m}\left(S i R^{\prime} i R^{\prime \prime}\right),
$$

所有上面的 $R^{\prime}$ 和 $R^{\prime \prime}$ 等价.

因此若条件 (2) 式成立, 则我们可消去所有 $\left(s_{i} \cdots\right)$ 形的排列，而不会把最优排列全消 光.

定理 2 若条件 (2) 式成立,则下面的条件必成立

$$
t_{1 q}(S i) \leqslant t_{1 q}\left(S_{j}\right)-a_{q i}+\min \left(a_{q i}, a_{q+1 i}, \cdots, a_{m i}\right), \quad 1 \leqslant q \leqslant m .
$$

显然条件 (4) 式若成立, 则条件 (B) 必然成立. 故由定理 2 可知消去准则 (A) 和 (B) 比 Szwarc 的消去准则 (2) 式要广泛.

定理 3 设 $i$ 和 $j$ 不属于序列 $S$, 序列集 $Q$ 是 $S$ 和 $i$ 以外的所有工件所排的序列的全 体. 若条件

$$
\begin{aligned}
t_{1 p}(S i) \leqslant \max \left\{t_{1 p}(S j)+\min _{r \geq q}\left[t_{q r}(i)-t_{q}(j)\right], B(S j \cdots)-\max _{R \in Q} t_{p m}(R)\right\}, \\
1 \leqslant p \leqslant q \leqslant m
\end{aligned}
$$

成立,则可消去所有 $(s j \cdots)$ 形的排列, 而不会把最优排列全消光.

定理 4 设序列 $S, s^{\prime}$ 和 $J$ 互不相交, $i$ 不属于 $s, S^{\prime}$ 和 $J$, 序列集 $Q$ 是 $s, s^{\prime}, J$ 和 $i$ 以 
外的所有工件所排的序列的全体. 若条件

$$
\begin{array}{r}
t_{1 p}(S i)+t_{q m}\left(J S^{\prime}\right) \leqslant \max \left\{t_{1 p}(S J)+A_{p q q}(i)+t_{q m}\left(S^{\prime}\right), B(S J \cdots)-\max _{R \in Q} t_{p q}(R)\right\}, \\
1 \leqslant p \leqslant q \leqslant m
\end{array}
$$

成立, 则可消去所有 $\left(s J \cdots s^{\prime}\right)$ 形的排列, 而不会把最优排列全消光.

$$
\text { 二、 } B\left(S \cdots S^{\prime}\right) \text { 和 } \max t_{p q}(\cdots) \text { 的计算 }
$$

更好地估计 $B\left(S \cdots S^{\prime}\right)$ 之值是提高消去法和分支定界法的效率的重要环节. 我们给出如 下公式: 设 $R$ 是 $S$ 和 $S^{\prime}$ 以外的所有工件的集, 根据 $a_{m \rightarrow 1}$ 和 $a_{m i}$ 之值, $j \in R$, 将 $R$ 中工件按照 Johnson 法则(见文献 [1]) 排成序列 $\left(j_{1} j_{2} \cdots i_{k}\right)$. 令

再定义

$$
\begin{array}{ll}
R_{1}=\left(j_{1} j_{2} \cdots i_{k} s^{\prime}\right), & R_{2}=\left(j_{2} i_{1} j_{3} \cdots j_{k} s^{\prime}\right), \\
R_{3}=\left(j_{3} j_{1} i_{2} j_{4} \cdots i_{k} s^{\prime}\right), \cdots, & R_{k}=\left(j_{k} j_{1} \cdots j_{k-1} s^{\prime}\right),
\end{array}
$$

$$
\begin{gathered}
b_{p}=t_{1 p}(S)+\max \left\{\min _{1 \leqslant \alpha \leqslant k}\left[t_{p m-2}\left(j_{\alpha}\right)+t_{m-1 m}\left(R_{\alpha}\right)\right], t_{p p}(R)+t_{p m}\left(S^{\prime}\right)\right\}, \\
1 \leqslant p \leqslant m-2, \\
b_{m-1}=t_{1 m-1}(S)+t_{m-1 m}\left(R_{1}\right), \\
b_{m}=t_{1 m}(S)+t_{m m}\left(R_{1}\right) .
\end{gathered}
$$

我们令

对于消去准则 (A) 和 (B)，因为有等式

$$
B\left(s \cdots S^{\prime}\right)=\max _{1 \leqslant p \leqslant m}\left\{b_{p}\right\} .
$$

$$
\max _{R \in Q} t_{q m}(j R)=\max _{q \leqslant r \leqslant m}\left\{t_{q r}(j)+\max _{R \in Q} t_{r_{m}}(R)\right\},
$$

所以只须研究 $\max _{\boldsymbol{Q}} t_{r m}(R)$ 的计算. 当 $R$ 包含大量工件时, 精确计算 $\max _{0} t_{r m}(R)$ 是很费时的, 我 们代之以它的上界. 设 $R=\left(j_{1} j_{2} \cdots j_{k}\right)$, 先在矩阵

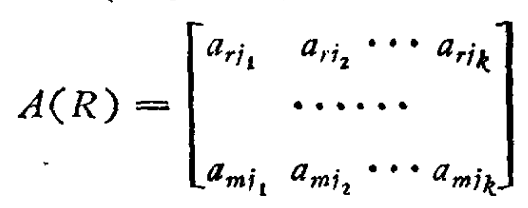

的每一列内任取一个最大数 $a_{i_{\gamma}}=\max _{r \leqslant p \leqslant m} a_{p i_{\alpha}}, \alpha=1, \cdots, k$. 将 $a_{i_{\alpha}}$ 从 $A(R)$ 中划去, 再在 $A(R)$ 的每一行余下的数中取出最大数 (空集的最大数为 0 ), 记为 $a_{r}, a_{r+1}, \cdots, q_{m}$, 则可以 证明

$$
\max _{Q} t_{r m}(R) \leqslant \sum_{\alpha=1}^{k} a_{i_{\alpha}}+\sum_{p=r}^{m} a_{p}-\min _{r \leqslant p \leqslant m}\left\{a_{p}\right\}
$$

\section{三、最优排列的算法}

每一个 $m \times n$ 排序问题都对应另一个 $m \times n$ 排序问题,称为逆问题. 逆问题的加工时间 矩阵为

$$
\left[\begin{array}{cccc}
a_{m n} & a_{m n-1} & \cdots & a_{m 1} \\
a_{m-1 n} & a_{m-1 n-1} & \cdots & a_{m-1} \\
& \cdots \cdots \cdots \cdots & \cdots & \\
a_{1 n} & a_{1 n-1} & \cdots & a_{11}
\end{array}\right] .
$$


容易证明: 逆问题的最优排列的逆排列就是原问题的最优排列. 下面我们把分支定界法和消 去法结合起来,给出一个计算最优排列的新方法.

(1) 先令 $S=S^{\prime}=\phi$, 令 $z^{*}=+\infty$, 通往 (2).

(2) 对正问题和逆问题用消去准则 (A)，(B)，(C)，(D) 进行检验、消去若干个分支 $\left\{\left(S j \cdots S^{\prime}\right)\right\}$ 或 $\left\{\left(s \cdots j S^{\prime}\right)\right\}$, 通往 (3).

（3）末消去的分支如只含两个排列，则分别计算各排列对应的总加工时间。令其中最小 者为 $z$. 取 $\min \left(z, z^{*}\right)$ 为新的上界 $z^{*}$ ，通往 (4); 如未消去的分支不只包含两个排列, 则计 算其下界 $B(\cdots)$, 通往 (5).

(4) 在树形分支图中每个未消去的分支所对应的下界中存在某下界 $B(\cdots)$, 它满足不 等式 $B(\cdots)<z^{*}$, 则通往 (5); 如每个未消去的分支所对应的下界都 $\geqslant z^{*}$, 则 $z^{*}$ 即为最短 的总加工时间, $z^{*}$ 对应的排列为最优排列,计算过程中止.

（5）在末消去的分支中取下界最小的分支 $\left\{\left(S_{1} \cdots S_{1}^{\prime}\right)\right\}$ 所对应的 $S_{1}$ 和 $S_{1}^{\prime}$ 作为新的 $S$ 和 $S^{\prime}$, 通往 (2).

\title{
今 考文祆
}

[1] Coffnian, Jr. E. G., Computer and Job-Shop Scheduling Theory, John Wiley \& Sons, 1976.

[2] 趝民义、韩继业，中国科学，1975，5: 462-470.

[ 3 ] Szwarc, W., Naval Res. Log. Quart., 18 (1971), 295-305.

[4] Szware, W., Opns. Res., 21 (1973), 1250-1259.

\section{A NEW METHOD FOR THE $m \times n$ FLOW-SHOP SEQUENCING PROBLEM}

\author{
Yue Min-yi (越民义) and Han Ji-ye（韩继业）
}

\begin{abstract}
In this paper a new algorithm is presented for solving the $m \times n$ flow-shop sequencing problem. First, we set down a few rules of elimination, and prove that one of them is more general than Szware's rules of elimination. Secondly, a new estimation formula of the branch and bound type is proposed. Our new algorithm is based on these results.
\end{abstract}

